\title{
Immunophenotyping of diffuse large B-cell lymphoma (DLBCL) defines multiple sub-groups of germinal centre-like tumours displaying different survival characteristics
}

\author{
JOHN J. ANDERSON ${ }^{1}$, SARAH FORDHAM ${ }^{1}$, LYNNE OVERMAN ${ }^{1}$, HELEN DIGNUM ${ }^{1}$, \\ KATRINA WOOD ${ }^{2}$, STEPHEN J. PROCTOR ${ }^{1}$, STEPHEN CROSIER ${ }^{1}$, BRIAN ANGUS ${ }^{2}$, \\ RACHEL E. CULPIN ${ }^{1}$ and TRYFONIA MAINOU-FOWLER ${ }^{1}$
}

${ }^{1}$ Department of Academic Haematology, Northern Institute for Cancer Research, University of Newcastle, NE2 4HH; ${ }^{2}$ Department of Pathology, The Royal Victoria Infirmary NHS Trust, UK

Received January 16, 2009; Accepted March 26, 2009

DOI: 10.3892/ijo_00000409

\begin{abstract}
Diffuse large B-cell lymphoma (DLBCL) forms a heterogeneous collection of aggressive non-Hodgkin's Lymphoma in which three principle classes of neoplasia have been defined according to gene expression and immunophenotyping studies. The present investigation sought to examine the immunophenotype of proposed subgroups and relate these to patient survival. A series of 155 DLBCL treated uniformly with anthracycline therapy in clinical trials, were stratified upon the basis of common biomarker expression with combination immunophenotype being related to patient overall survival. Stratification of tumours with respect to combined expression profiles of the three biological markers (CD10, Bcl-6 and MUM-1) revealed six groups showing significant differences in survival $(\mathrm{p}=0.014)$. The greatest difference resided between distinct populations of germinal centre (GC) cell tumours; the first being $\mathrm{CD} 10^{-}, \mathrm{Bcl}-6^{+}$, MUM-1- and the second CD10 ${ }^{+} \mathrm{Bcl}-6^{+} \mathrm{MUM}^{-} 1^{+}(\mathrm{p}=0.002)$. The former group displayed median survival time of 143 months, the latter only 11 months. A third population of GC tumours (CD10+ $\mathrm{Bcl}^{-6^{+}}$and $\left.\mathrm{MUM}-1^{-}\right)$also displayed a relative short median survival (32 months). Of the three groups presenting a non-GC or activated $\mathrm{B}$ cell $(\mathrm{NGC} /$ $\mathrm{ABC})$ phenotype, only one $\left(\mathrm{CD} 10^{-}, \mathrm{Bcl}-6^{+}\right.$and $\left.\mathrm{MUM}-1^{+}\right)$ presented short-term median survival (27 months) comparable with poor prognosis GC sub-populations. Within the remaining ABC tumour groups (CD10- Bcl-6- MUM-1- and CD10Bcl-6- MUM-1 $\left.^{+}\right)$patients presented intermediate median survival times of 54 and 58 months, respectively. Thus, the GC phenotype did not act as a universal indicator of good clinical prognosis, but rather multiple groups of GC tumours were
\end{abstract}

Correspondence to: Dr John J. Anderson, Academic Haematology, The University of Newcastle, Newcastle-upon-Tyne, NE2 4HH, UK E-mail: j.j.anderson@ncl.ac.uk

Key words: diffuse large B-cell lymphoma, high grade nonHodgkin's lymphoma, immunophenotyping associated with distinct overall survival profiles. Ultimately, the data allowed definition of a predictive algorithm defining three groups predicting poor, intermediate and good clinical prognosis. The first of these comprised two patient subpopulations with GC-like tumours together with one subpopulation of $\mathrm{NGC/ABC}$, the second two sub-populations of ABC-like tumours, and the final a single group of GC-like tumours associated with optimal long-term survival.

\section{Introduction}

Diffuse large B-cell lymphoma (DLBCL) represents a heterogeneous group of aggressive non-Hodgkin's lymphomas (NHL) accounting for about $40 \%$ of such tumours (1).

Management of DLBCL presents an ongoing problem, with therapy centring upon variations of anthracycline-based combination chemotherapy the most common forms being variations on the 'CHOP' regime. Core therapy has been augmented by intensification regimes, autologous bone marrow and stem cell transplantation protocols. Treatment response, however, has been improved considerably in resent years by the additional application of the CD20-specific monoclonal antibody Rituximab (R). This R-CHOP regime represents the present optimal management regime for DLBCL. However, despite these advances, long-term survival still remains poor and many patients relapse failing to respond to either primary or supplementary therapies.

Direction of therapy and prediction of outcome at the time of presentation remains difficult and is, in the greater part, still dependent upon the 'International Prognostic Index (IPI)', proposed in 1993 (2). The IPI is based on five clinical features known to be risk factors in DLBCL (age, tumour stage, serum lactate dehydrogenase concentration, performance status and number of extra-nodal disease sites). This however, retains a considerable degree of subjectivity and substantive efforts in research have been directed towards the ultimate improvement of this index and/or towards the definition of alternative methods, which may be used to evaluate risk and clinical outcome. Toward this end, many studies have sought to qualitatively, quantitatively or semi-quantitatively assess the impact that evaluation of expression of a variety of 
biological markers may have upon these objectives (3-16). The most extensive of these studies have analysed genome wide RNA expression, utilising cDNA micro-arrays. Attaining intra-laboratory reproducibility, defining consensus of panels of prognostically significant biomarkers, has proven a difficult task. However, array-based studies have reinforced the definition of three broad groups of DLBCL based upon characteristic expression profiles; these have been related to 'germinal centre (GC) cell', 'activated B cell (ABC) or nongerminal centre cell (NGC)' or primary mediastinal lymphoma (PML) phenotypes.

Currently, a number of proteomic-based studies have sought to corroborate and supplement the findings of arraybased studies. Much of this work has employed immunohistochemical studies of protein expression, in sections of formalin-fixed paraffin wax-embedded tissue. Algorithms defining groups of patients presenting significant differences in survival have been developed, by considering expression of principally three proteins, namely: the neutral membrane metallo-endopeptidase: CD10, the cellular oncogene Bcl-6 and the $\mathrm{B}$ cell differentiation marker multiple myeloma oncogene MUM-1/IRF-4. These algorithms have proposed a simple means of defining $\mathrm{GC}$ and $\mathrm{ABC}$ subsets, potentially predicting tumour behaviour and patient prognosis at the time of diagnosis. However, given the variability of the projected prognostic significance of CD10 expression in these tumours, the present study reviewed the efficacy of these current algorithms with respect to predicting patient prognosis. Furthermore, we sought to assess the contribution of expression of two anti-apoptotic proteins, IAP-4 and Bcl-2, in stratification of DLBCL. It is hoped that ultimately, the growing body of evidence from such studies may help more clearly define factors which may augment current means of stratifying DLBCL. In addition, they may also aid in determining prognosis and prediction of resistance to combination chemotherapy thereby facilitating more robust risk stratification and improved patient management.

\section{Materials and methods}

Study population. A cohort of 155 patients was drawn from 436 patients originally enrolled in trials implemented by the Scottish and Newcastle Lymphoma Group and diagnosed with DLBCL. Diagnosis was based upon Revised EuropeanAmerican Lymphoma (REAL) criteria (17). Patients received treatment in the form of conventional ' $\mathrm{CHOP}$ ' combination chemotherapy, in line with trial protocols.

Of the patient cohort, $73(47.1 \%)$ were female and 82 $(52.9 \%)$ were male. Patient age range varied from 16 to 79 years with a mean age at diagnosis of 53.46 years ( $\mathrm{SD} \pm 13.8$ years). Of the entire cohort, $87.1 \%$ presented with tumours of primary nodal origin and $12.9 \%$ extra-nodal origin. The mean survival time, irrespective of the treatment, was only 63.14 months from the time of diagnosis. For the patients who had complete IPI data, $35.4 \%$ were assigned to the low risk group, $30.8 \%$ to the low intermediate, $29.2 \%$ to the high intermediate and $4.6 \%$ to the high-risk group. At the close of the trials, $63.2 \%$ patients had died, $35.5 \%$ remained alive and $1.3 \%$ were censored as they had either left the trial or died of causes other than DLBCL.
Tissue sections. All tissue samples were fixed and embedded in accordance with standard laboratory protocols. Subsequently, $3 \mu \mathrm{m}$ tissue sections were mounted on Super-frost plus slides. Two Haemato-pathologists independently examined H\&E stained tumour sections, critically reviewing each case and assigning diagnosis.

Immunohistochemistry (IHC). Tumour sections were stained with monoclonal antibodies against: CD10, Bcl-6, Bcl-2, IAP-4 and MUM-1. Immuno-staining with CD10, Bcl-6, Bcl-2 (Novocastra Laboratories, UK), MUM-1 (Dako Laboratories, UK) and IAP-4 (JJA) (18), was performed in accordance with standard immunohistochemical protocols utilising predetermined optimal primary antibody concentrations, SAB detection, and 3'3'-di-aminobenzidine tetra-hydrochloride (DAB) as chromagen. Dual immunostaining with analyte specific antibody/CD79a pan B cell-specific antibody was performed in a similar fashion, using a Ventana Benchmark ${ }^{\mathrm{TM}}$ System (Ventana Medical Systems, Tucson, AZ). However, in 'double staining procedures', iView DAB' chromagen, which in all other cases was used to detect the primary target analyte (CD10, Bcl-6, Bcl-2 IAP-4 and MUM-1) was used to detect binding of CD79a antibody to its membrane associated target, while the 'Enhanced Ventana Red Alkaline Phosphatase' detection system was employed to visualise nuclear bound antibody. In all runs, tonsil sections served as controls.

Scoring. In analyses of IHC results 'cut-off points' defining the positivity of individual analytes were established based upon scoring systems in which 250 cells were examined and graded and the percentage of cells staining was recorded: no staining was graded as negative, while significant staining was graded on an ascending three point scale (1-3). The cut off point defining positivity of tumour expression of CD10, Bcl-6 and Bcl-2 was set as $1+$ staining being evident in $20 \%$ or more of tumour cells examined, consistent with published studies. MUM-1 expression in tumours was considered positive when $40 \%$ or more of cells showed evidence of nuclear staining. Thus, in each of the above groups, 'negative tumours' defined in this fashion can be more accurately considered as low expression groups, rather than categorically negative. IAP-4 tumour expression was optimally defined applying a scoring system analogous to that of the Detre system. This gave a range of values between 1 and 300, with a median value of 150 . Thus, two groups of tumours were defined and distributed either side of the median. Finally, in Ki-67 expression, high expression was considered as $\geq 95 \%$ of cells showing significant staining. Scoring was again carried out independently by two observers and disparities resolved by discussion.

Statistical analysis. Survival analyses were performed using both the univariate Kaplan-Meier (KM) method (19) and the Cox regression (CR) model (20), utilizing the Log Rank (LR) statistic as the comparator statistic. SPSS statistical software v11.04 (SPSS, Inc., Chicago, IL, USA) was employed to perform all analyses.

Ethics approval for the study. The study was performed under the sponsorship of The Marrow and Stem Cell 2000 Millennium Fund, with samples being drawn from under the 
auspices of the Scottish and Newcastle Lymphoma Group Clinical trials NHL-V and Va.

\section{Results}

The International Prognostic Index as relating to the study cohort. The clinicopathological characteristics of the patient cohort for which data were available (age, extra-nodal involvement, etc) are described in 'Materials and methods'. No significant difference in patient survival time between patients with nodal $(87.1 \%)$ or extra-nodal tumours $(12.9 \%)$ was observed (KM; $\mathrm{p}=0.8639)$. Complete data to formulate IPI categories were limited in the population, encompassing only 62 of 155 patients. However, within this sub-set of patients the IPI proved effective for predicting survivorship $(\mathrm{KM} ; \mathrm{p}<0.0001)$ in an unsegregated population, with median survival times of 114, 74, 24 months and 1 month being evident for IPI groups 1, 2, 3 and 4, respectively (Fig. 1).

Immunohistochemical staining and confirmation of B cell homogeneity of examined lymph nodes. Immunostaining with each of the primary antibodies applied in this study, in conjunction with the 'Ventana iView DAB chromagen', is shown in Fig. 2A-F. Sections of DLBCL revealed sheets of tumour cells, which morphologically appeared 'homogeneous'. To help ensure that these 'homogeneous' cell sheets were predominantly B-cells, subsets of samples were double stained using CD79a pan B cell-specific antibody, as well as other analyte specific antibodies. An example is given in Fig. 2G, which demonstrates dual staining achieved with CD79a and MUM-1 specific antibodies. In every instance, tumour cells were found to express cell membraneassociated CD79a, while a variable percentage of tumour cells presented nuclear MUM-1 staining. However, and importantly, although CD79a $\mathrm{a}^{+} \mathrm{MUM}-1^{+}$and CD79a $\mathrm{a}^{+}$MUM-1 tumour cells were clearly evident, no CD79a-MUM-1 ${ }^{+}$ tumour cells were apparent (Fig. 2, Panel G). This supported the B-cell lineage of 'scored' tumour cells, irrespective of their capacity to express MUM-1 or other primary analytes. This pattern of immunostaining also held true irrespective of whether the tumour was nodal or extra-nodal in origin.

CD10, Bcl-2, IAP-4 and MUM-1, but neither Bcl-6 nor Ki-67 served as markers of significant prognostic value in DLBCL. Analysis of the frequency of expression of each of the primary analytes within our cohort, indicated that positive/high expression of CD10 was observed in $35 \%$ of patient tumours (53 of 152), Bcl-2 in 58\% (90 of 154), IAP-4 in 22\% (33 of 150 ), MUM-1 in 34\% (52 of 154), and Bcl-6 in 84\% (128 of 152). The results of individual Kaplan-Meier survival analyses are presented in Fig. 3. Positive/high expression of CD10, MUM-1 and the anti-apoptotic proteins Bcl-2 and IAP-4 each served as significant indicators of reduced long-term survival and increased the relative risk (Fig. 3). The strongest individual independent marker of poor clinical prognosis proved to be elevated $\mathrm{Bcl}-2$ expression ( $\mathrm{p}=0.0025)$. This was marked by comparative median survival times of 121 and 24 months in low and high expressing patient tumour groups, respectively (KM; $\mathrm{p}=0.0025$; $\mathrm{CR}$ : relative risk $(R R)=1.9)$ Fig. 3B. In contrast to each of the other analytes

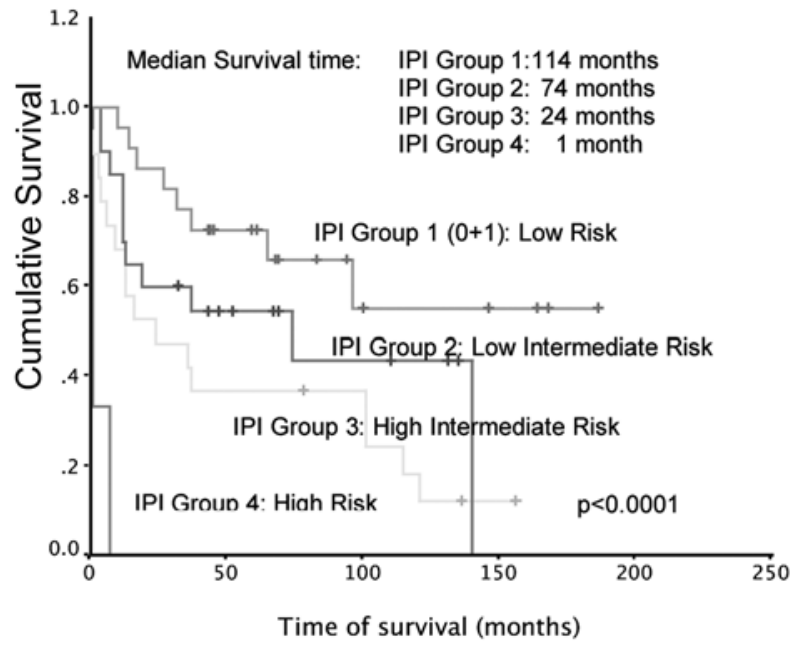

Figure 1. International Prognostic Index I.P.I related to overall survival in that portion of the study cohort for which complete IPI data were available (64 of 155 patients).

examined, neither high expression of the Bcl-6 oncogene product $(\mathrm{p}=0.381)$, nor Ki-67 (0.09), presented any significant predictive value.

CD10 and Bcl-6 immunostaining applied in defining Germinal Centre (GC) phenotype to predict patient prognosis and management. Considering CD10 and Bcl-6 expression together, as the once conventional classical defining criteria of GC phenotype, 53 tumours were shown to possess this phenotype (either $\mathrm{CD} 10^{+} \mathrm{Bcl}-6^{+}$or $\mathrm{CD} 10^{+} \mathrm{Bcl}-6^{-}$), while 93 proved to be of the classical NGC phenotype (either CD10- Bcl- $^{+}{ }^{+}$or CD10- Bcl-6 $^{-}$). Based upon this break down of our population, it was evident that GC cell phenotype, defined and weighted essentially by CD10 positivity, in this instance acted as an indicator of poor prognosis. Those patients with GC type tumours displayed significantly reduced median survival time of 24 months when compared with the remaining patients with NGC tumours who presented a median survival time of 84 months (KM; $\mathrm{p}=0.0164)$.

Immunophenotyping applied in decision/prediction algorithms. Application of CD10/Bcl-6/MUM-1 algorithms and proposal of a third algorithm weighted on survival. Two 'decision algorithms' defining cell phenotype and projecting patient prognosis have to-date been proposed by individual research groups (Fig. 4A and B) $(10,21)$. Applying the first of these algorithms (Algorithm A) to our cohort: tumours of 53 patients $(33.7 \%)$ presented the $\mathrm{GC}-\mathrm{CD} 10^{+}$phenotype, 51 $(33.37 \%)$ the GC-CD10- Bcl- $^{+}$MUM-1- phenotype, 25 $(16.5 \%)$ the NGC/ABC-CD10- $\mathrm{Bcl}-6^{+}$MUM- $1^{+}$phenotype and $22(14.56 \%)$ the NGC/ABC-CD10- Bcl-6 phenotype. A significant difference was observed between the long-term survival of these groups (KM; p=0.0088) (Fig. 5A). Most markedly, the two GC cell groups defined by this algorithm showed distinctly different prognoses (KM; $\mathrm{p}=0.0026$ analysis being performed pair-wise between strata). Patients whose tumours were $\mathrm{CD} 10^{+}$showed extremely poor survival, with a median survival time of $24 \pm 8.64$ months. In contrast, patients 

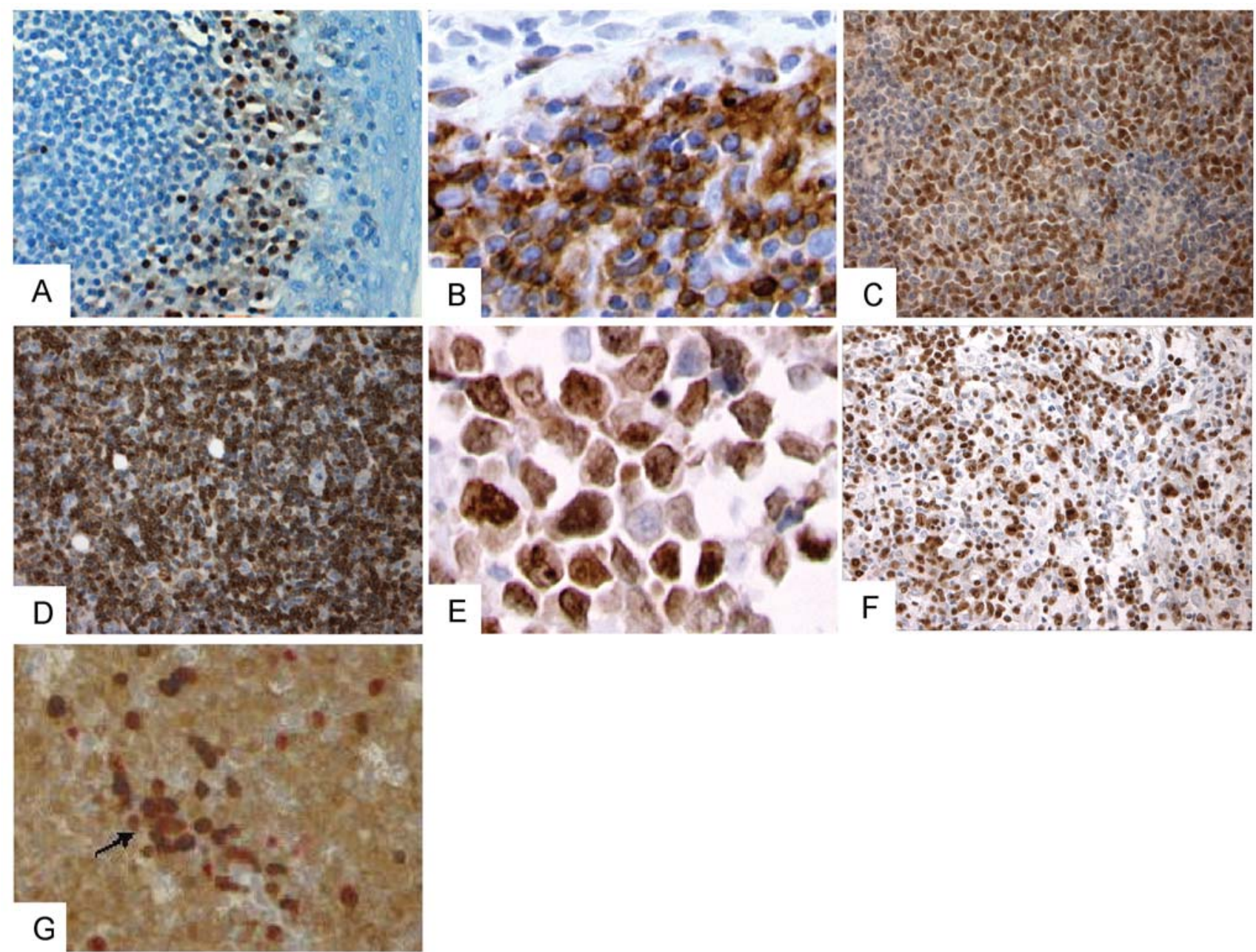

Figure 2. Results of immunohistochemical staining (IHC) of normal tonsil and DLBCL tissue with each analyte-specific antibody. (A) Immunohistochemical staining of healthy tonsil with MUM1p antibody haematoxylin counter-stain. MUM-1 expression was observed in a small proportion of cells within the central area of germinal centers of normal tonsil control tissue. However, as predicted with a 'later stage differentiation marker' staining with MUM-1 predominated in centrocytes in the outer 'light zone' of this type of tissue. (B-F) DLBCL, positive IHC staining with CD10, Bcl-6, Bcl-2, IAP-4 and Ki-67 respectively. (G) Dual IHC staining of DLBCL with MUM-1 antibody (red nuclear staining) and CD79a antibody (brown membrane staining); haematoxylin counter-stain. The arrow indicates a cluster of CD79a $\mathrm{a}^{+} \mathrm{MUM}-1^{+}$tumour cells. The images were captured using an Olympus BX60 microscope (Olympus, UK) and acquired using Image Pro Express software (Media Cybernetics Inc.).

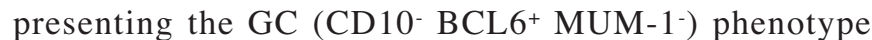
showed the longest median survival time observed of 143 months. Patients showing high expression of Bcl-6 as well as expression of MUM-1 [NGC/ABC (CD10- BCL6 $^{+}$MUM-1 $\left.^{+}\right)$] showed similar survival to the $\mathrm{CD} 10^{+} \mathrm{GC}$ group, presenting a median survival time of $27 \pm 10.62$ months (KM; $\mathrm{p}=0.9818$ ), while those patients whose tumours showed neither expression of CD10 nor Bcl-6 presented a median survival time of 58 months.

Applying the second widely cited algorithm (Algorithm B: Fig. 4B) to our cohort, again indicated overall a significant difference in survival between defined groups of patients (KM; $\mathrm{p}=0.0049$ ) (Fig. 5B). Once more, two apparently distinct groups of GC B cell-like tumours were clearly apparent (KM; $\mathrm{P}=0.0023$ ). The first being the $\mathrm{CD} 10^{+}$group described previously showing rapid decline, and a second group (CD10- MUM-1 $^{-}$) showing good long-term survival (median survival time $=128$ months). The defined ABC group $\left(\mathrm{CD} 10^{-}\right.$ MUM-1 $1^{+}$) confirmed the predictive power of MUM-1+ expression, with patients displaying characteristically short- term survival (median survival time $=30 \pm 10$ months). However, it was notable that no significant difference was apparent between the survival of the NGC/ABC group and that of the $\mathrm{CD} 10^{+} \mathrm{GC}$ cell group (KM; $\left.=0.6483\right)$. As would therefore be predicted, survival of this NGC/ABC group also proved significantly different from that of patients with CD10- MUM-1- GC tumours (KM performed pair-wise between strata; $\mathrm{p}=0.0125$ ) (Fig. 5B). This data further supported the prognostic power of MUM-1 expression, particularly in the apparent absence of CD10 expression.

The need to consider each potential IHC phenotype closely led us to propose a third algorithm (Algorithm C), inclusively classifying tumours with respect to expression profiles of all three widely applied biological markers (i.e. CD10, Bcl-6 and MUM-1). Theoretically, this could potentially define eight groups. In practice, six groups were apparent (Fig. 5C). All CD10+ tumours proved to be $\mathrm{Bcl}-6^{+}$, reenforcing their classification as $\mathrm{GC}$ cell tumours. No $\mathrm{CD} 10^{+}$ Bcl-6- tumours were identified. Applying KM survival analysis, utilising the LR test pooled across the strata (six groups), 
A

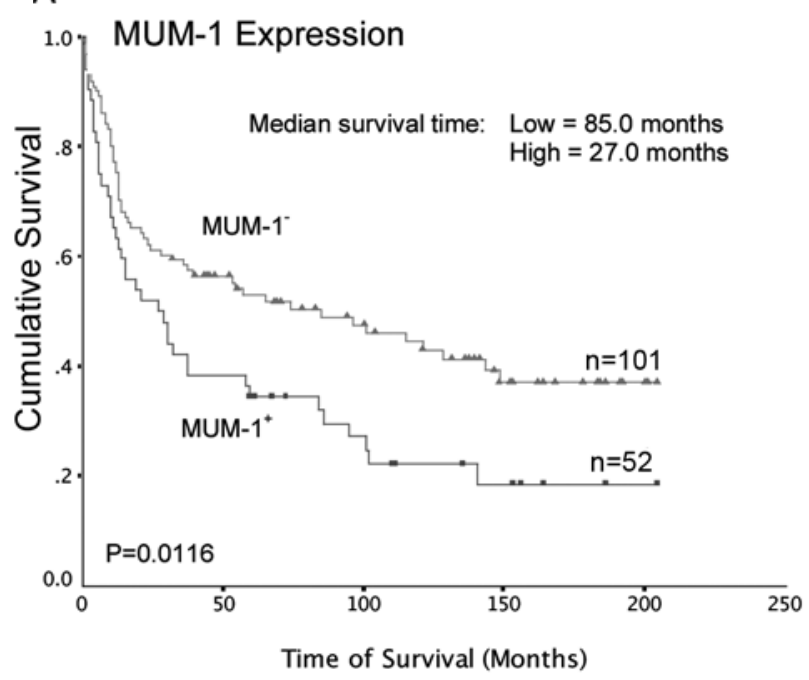

C

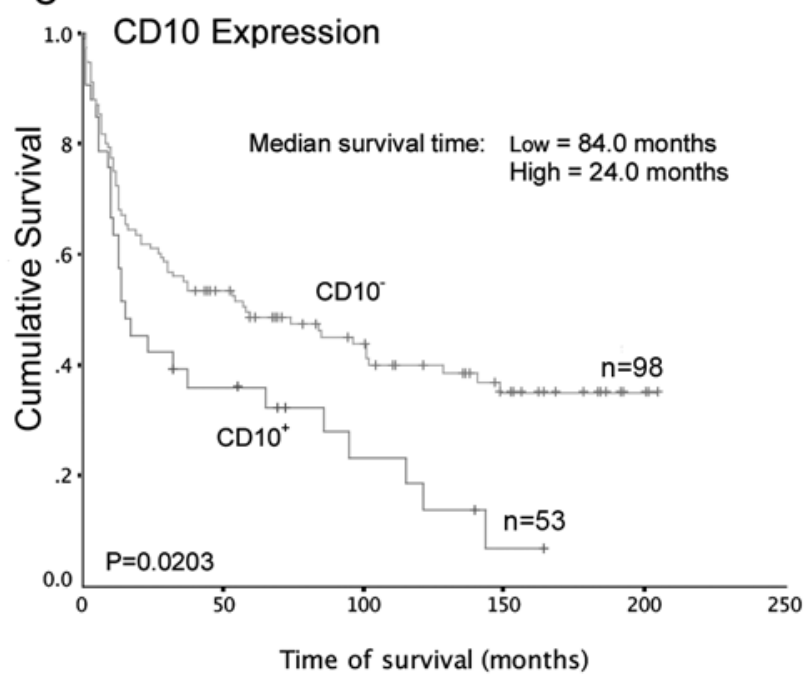

B

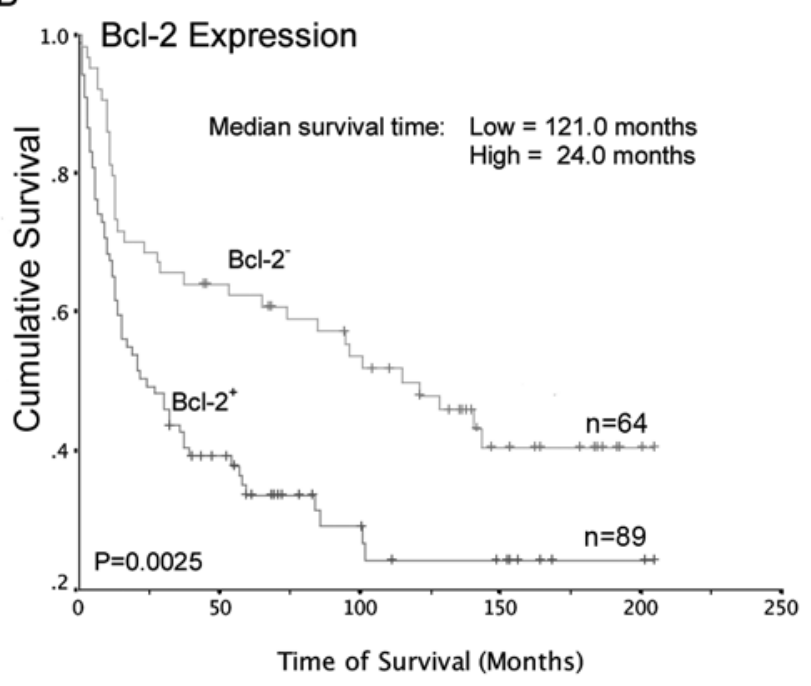

D

1.0 IAP-4 Expression

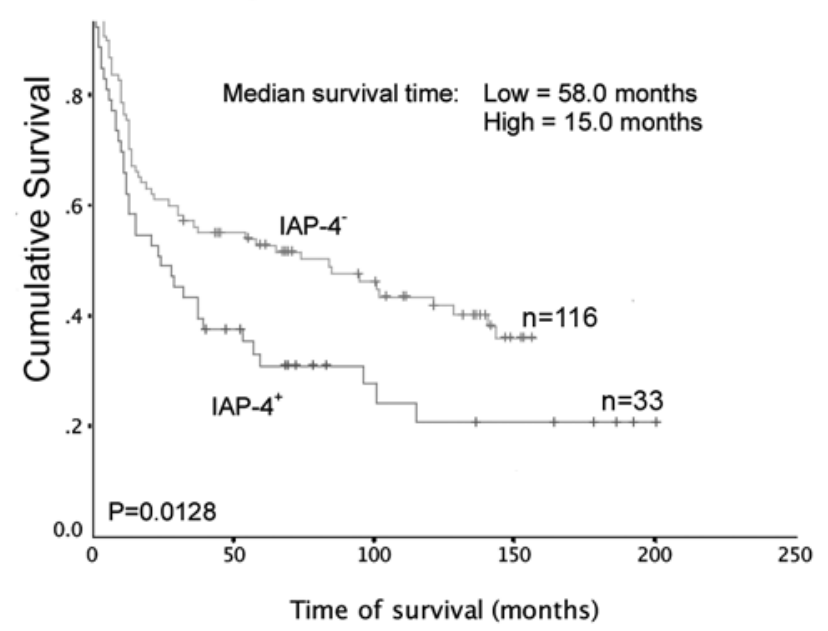

Figure 3. Relationship of MUM-1, Bcl-2, CD10 and IAP-4 expression to survival of patients with DLBCL: Kaplan-Meier analysis demonstrates that high levels of expression of MUM-1, Bcl-2, IAP-4 and CD10 are each associated with reduced survival within the study cohort with the greatest prognostic power appearing to reside with expression of $\mathrm{Bcl}-2$.

indicated a significant difference in survival between subpopulations $(\mathrm{p}=0.014)$. However, reviewing the comparator statistic pair-wise between groups, revealed that this difference resided ostensibly with the comparison of particular paired populations. The greatest difference in survival again resided between distinct populations of patients with GC cell tumours. Specifically CD $10^{+} \mathrm{Bcl}-6^{+}$MUM-1- and CD $10^{+}$ Bcl- $6^{+}$MUM- $1^{+}$tumours ( $\left.\mathrm{p}=0.002\right)$. Similarly, a significant but smaller difference in overall median survival was observed between patients with $\mathrm{CD}^{-} 0^{-} \mathrm{Bcl}-6^{+} \mathrm{MUM}-1^{-}$and CD10+ Bcl-6+ MUM-1- GC-like tumours ( $\mathrm{p}=0.0164$ ). Furthermore, a substantive difference in survival was observed between patients with $\mathrm{CD} 10^{-} \mathrm{Bcl}-6^{+}$MUM-1- GC tumours and CD10- $\mathrm{Bcl}-6^{+}$MUM-1+ ${ }^{+}$NGC/ABC tumours ( $\mathrm{p}=0.0073$ ). Scrutiny of the data suggested that overall these six groups could be reduced on the basis of their survivorship into three groups. Thus, algorithm $\mathrm{C}$ is proposed, defining three groups in respect to overall survival: i.e. those patients showing good, intermediate or poor prognosis (Fig. 4C). Kaplan-Meier analysis (Fig. 5D) showed a strong significant difference between the survival of these groups (KM with LR pooled over the strata, $\mathrm{p}=0.0036$ ). Clearly the greatest difference lay between good and poor prognosis groups, where the LR value was equal to 10.47 , equating with a p-value $=0.0012$. Those patients exhibiting poor prognosis presented $\mathrm{CD} 10^{+}$ Bcl-6+ GC tumours as well NGC/ABC tumours which were Bcl-6 $6^{+}$MUM-1+ but CD10-. Among these patient groups, median survival time varied between 11 and 32 months with a combined median survival time of 27 months. Tumours from patients exhibiting 'intermediate' survival appeared to comprise those lymphomas defined as $\mathrm{NGC/ABC}$ and $\mathrm{ABC}$ tumours, which were both $\mathrm{CD}^{-} 0^{-}$and $\mathrm{Bcl}-6^{-}$irrespective of their MUM-1 expression status. Independent median survival times in these groups varied between 54 and 58 months, while their combined overall median survival time was 56 months. Finally, optimum long-term survival was observed in those patients whose tumours were defined as comprising GC cells, which were $\mathrm{Bcl}-6^{+}$but expressed neither CD10 nor MUM-1. This group of patients showed an overall survival time of 143 months, representing in 
A Algorithm A

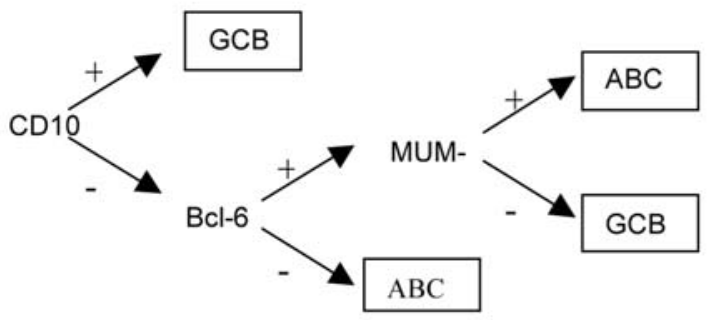

B Algorithm B

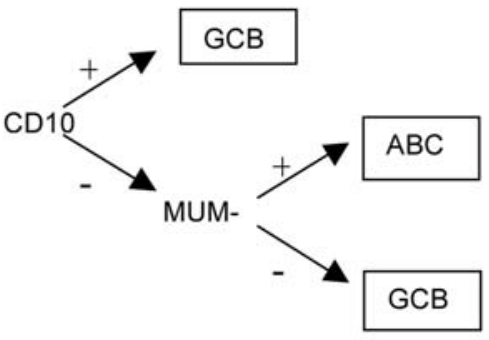

C Algorithm C

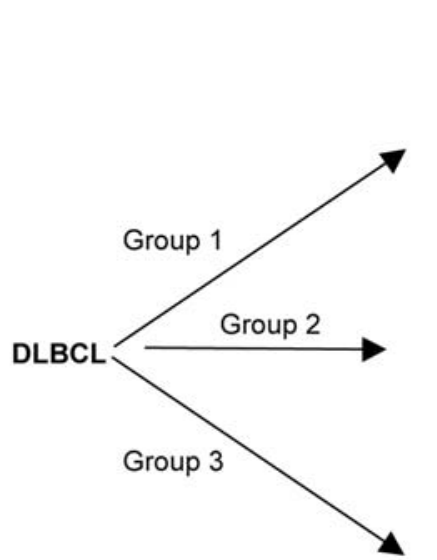

GCB: CD10 ${ }^{+}$Bcl- $6^{+} \mathrm{MUM}^{+} 1^{+} 11$ months $(n=12)$

GCB: CD10 ${ }^{+}$Bcl- $6^{+}$MUM-1- 32 months $(n=40)$

NGC/ABC: CD10 $\mathrm{Bcl}^{-} 6^{+} \mathrm{MUM}^{-1^{+}} 27$ months $(n=25)$
PROGNOSIS

\section{Poor}

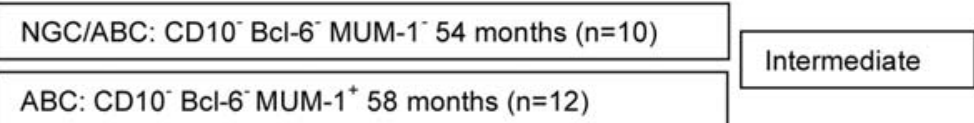

GCB: CD10 ${ }^{-} \mathrm{Bcl}-6^{+}$MUM-1 143 months $(n=50)$

Good

Figure 4. Predictive IHC algorithms stratifying DLBCL with respect to clinical prognosis.

excess of a 10-fold increase in survival time over those patients whose tumours showed expression of Bcl-6, CD10 and MUM-1.

Differentially defined Germinal Centre $(G C)$ tumour subgroups and expression of the anti-apoptotic proteins; BCL-2 and IAP-4. Bcl-2 and IAP-4 were each found to be two of the three individual biomarkers most strongly associated with patient survival (Fig. 3). Co-expression of these analytes enhanced their individual predictive power. Kaplan-Meier analysis across the strata indicated a significant difference between the four defined subgroups (Fig. 6A) $(p=0.0027)$, while analysis between individual strata indicated that median survival time declined progressively with expression of IAP-4 and Bcl-2. Patients with Bcl-2- IAP-4- tumours displayed a median survival time of 140 months. However, this was reduced in patients with $\mathrm{Bcl}-2^{-} \mathrm{IAP}-4^{+}$tumours to 37 months $(p=0.0202)$, in those with Bcl-2+ IAP-4- tumours to 30 months $(\mathrm{p}=0.0101)$, and finally in those with tumours co-expressing Bcl-2 and IAP-4 to only 14 months. This represented a strong association of co-expression with poor clinical prognosis when compared to expression of either marker alone $(\mathrm{p}=0.0005)$.

Among all tumours presenting a GC phenotype, Bcl-2 expression was again associated with reduced patient survival (LR pooled across strata; $\mathrm{p}=0.0071$ ) (Fig. 6B).
Within the poor prognosis (PP)-GC group, median survival was further reduced from the composite median of 24 months to only 15 months. Conversely, median survival in patients with Bcl-2-PP-GC tumours rose to 29 months. Within the good prognosis (GP)-GC group, patients whose tumours were $\mathrm{Bcl}-2^{+}$displayed a median survival of 36 months, clearly delineating them from their Bcl-2- counterparts (median survival $=143$ months). Analysis of IAP-4 expression in GC tumours once more revealed significant survival differences between subgroups ( $\mathrm{p}=0.0078$ ) (Fig. 6C). Within the PP-GC sub-population, IAP- $4^{+}$expression was again associated with reduced median survival time (13 months). Comparatively, patients with GP-GC IAP- $4^{+}$tumours presented a median survival time of 64 months, while their IAP-4- counterparts displayed a median survival time of 122 months. Unfortunately, restricted sample numbers precluded meaningful analysis of co-expression of Bcl-2 and IAP-4 in the defined GC subgroups.

Differentially defined GC subgroups and additional clinical parameters. No significant difference was apparent in survival, within GP-GC and PP-GC tumour subgroups when segregated on the basis on nodal or extranodal presentation. Similarly, age at presentation, which was clearly an indicator of prognosis across the entire cohort, $(p=0.003$, patients $\geq 60$ median survival of 16 months c.f. $<60$ median survival 
A

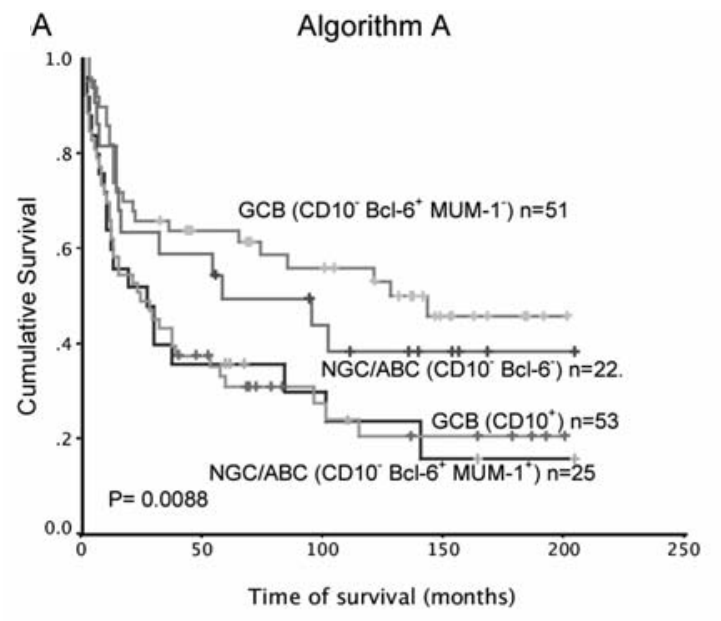

Median survival times:

NGC/ABC (CD10- BCL6+ MUM1+) NGC/ABC (CD10- BCL6-) GCB (CD10+) GCB (CD10- BCL6+ MUM-1-)

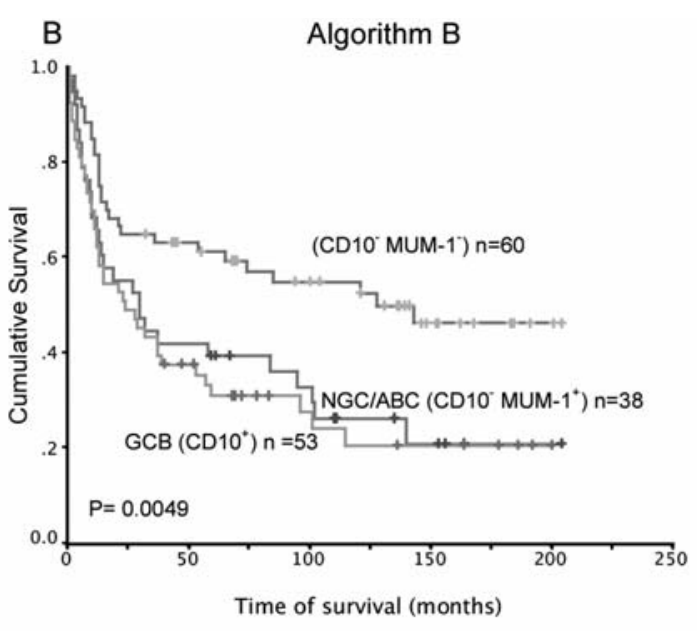

Median survival times:

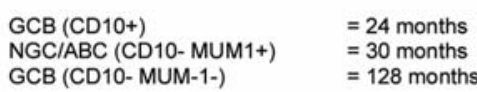

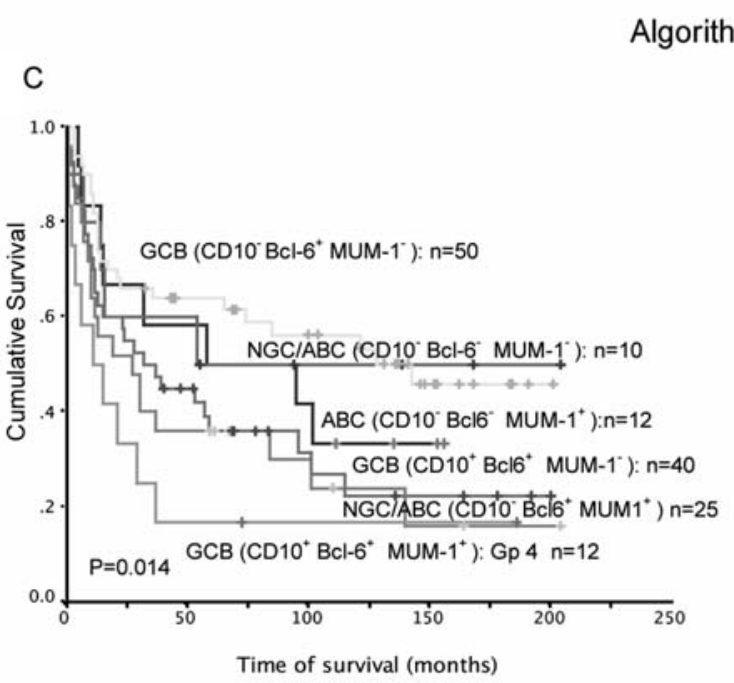

Algorithm C

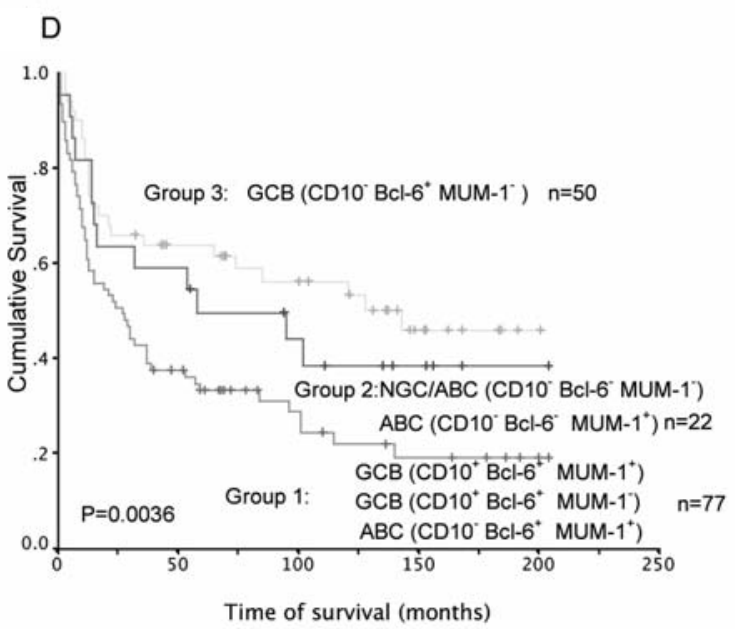

Median survival times

Poor prognosis group

$=27$ months

Intermediate prognosis group $\quad=56$ months

Good prognosis group

= 143 months

Figure 5. Survival of patients presented in the context of decision algorithms.

of 86 months), remained a significant indicator of prognosis within both the defined GC cell groups $(p=0.0004)$. Age appeared of greater prognostic strength within the GP-GC group ( $\mathrm{p}=0.007$ : median survival time patients $\geq 60=36$ months c.f. $<60=124$ months) as compared to the PP-GC group ( $\mathrm{p}=0.034$ : median survival time patients $\geq 60=12$ months c.f. $<60=32$ months).

\section{Discussion}

Many studies have sought to evaluate the potential of a wide variety of proteins as objective biomarkers in DLBCL, applying immunohistochemical techniques. Candidates have included cell cycle regulatory molecules, lymphokines and cytokines, differentiation associated markers, apoptosis-related gene products, growth regulatory and growth signal respon- siveness molecules, as well as molecules involved in tissue invasion and tumour angiogenesis (22). Key molecules have been documented as central players in the genesis and progression of DLBCL, especially those whose expression and/or activation is governed directly as a result of 'pathologically' significant chromosome translocation or somatic mutation. Such normally tightly regulated products include the oncogene/transcriptional repressor Bcl-6, the anti-apoptotic protein Bcl-2 as well as the pro-proliferative oncogenic transcription factor c-Myc. Expression of each may be ameliorated by translocation and mutational events, occurring during maturation of the immunoglobulin gene locus.

The current investigation of the application of IHC immuno-profiling of DLBCL in the pre-retuximab era indicated surprisingly, that Bcl-6 over-expression did not appear to relate to clinical prognosis. This was somewhat 
A

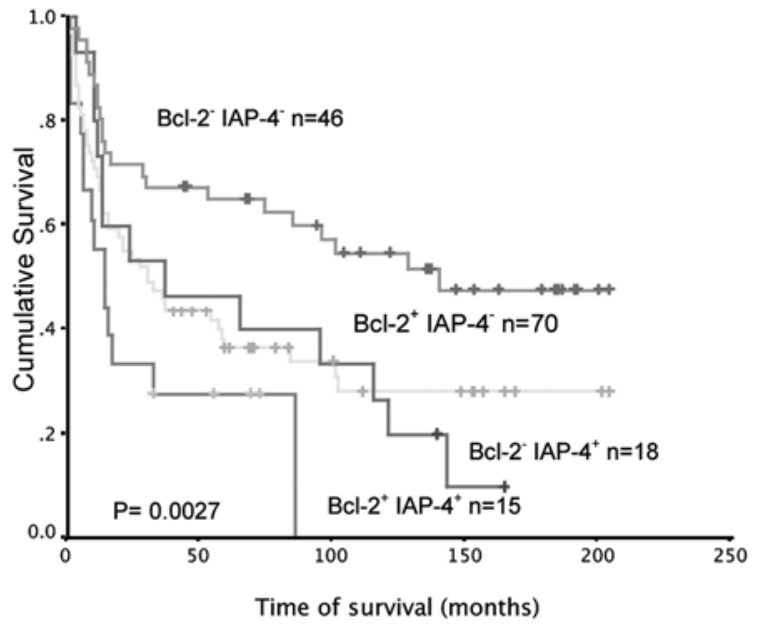

Median Survival times:

Bcl-2 IAP-4": 140 months

Bcl-2 ${ }^{+}$IAP-4: 30 months

Bcl-2 IAP-4 $4^{+}: 37$ months

Bcl-2 ${ }^{+}$IAP $-4^{+}$: 14 months

B

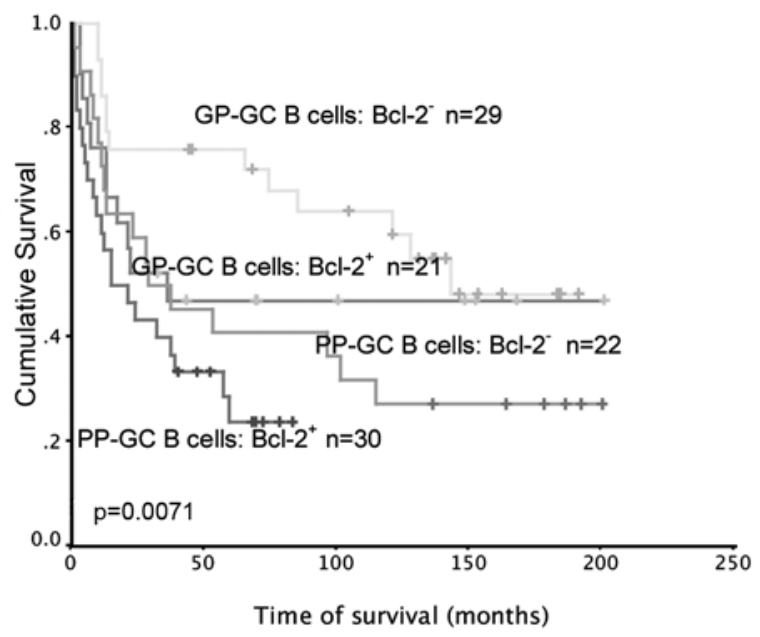

Median Survival times:

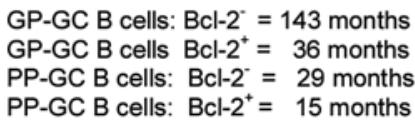

C

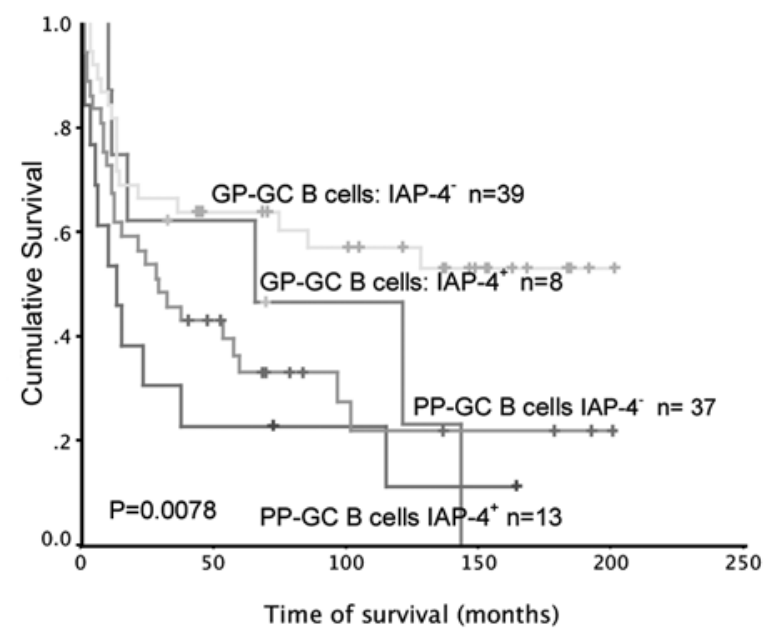

Median Survival times :

GP-GC B cells: IAP-4 $=122$ months

GP-GC B cells IAP- $4^{+}=65$ months

PP-GC B cells: IAP-4 $=29$ months

PP-GC B cells: IAP- $4^{+}=13$ months

Figure 6. Survival analysis of anti-apoptotic protein expression in patients with DLBCL with reference to (A) the entire study population and (B and C) and specifically patients whose tumours stratify into Good Prognosis-(GP)-GC (CD10- $\mathrm{Bcl}-6^{+}$MUM-1 $)$and Poor Prognosis (PP)-GC (CD10 ${ }^{+}$Bcl-6 ${ }^{+}$MUM- $\left.1^{+}\right) /$ $\left(\mathrm{CD} 10^{+} \mathrm{Bcl-6}{ }^{+} \mathrm{MUM}-1^{-}\right)$tumour immunophenotypes.

unexpected, given the role of this product in germinal centre development, affinity maturation, and its ability to interact differentially with different co-factors regulating distinct biological pathways (23). However, IHC studies reporting both negative and positive associations of BCL-6 expression with overall patient survival have been published $(7,24)$. Similarly, in our cohort Ki67 also failed to show any association with clinical outcome. Once more however, reports vary with respect to the relationship of IHC assayed
Ki-67 over-expression and patient overall or event free survival. In contrast, tumour expression of high levels of anti-apoptotic proteins Bcl-2 ( $\mathrm{p}=0.0025)$, IAP-4 ( $\mathrm{p}=0.0128)$ or MUM-1 ( $p=0.0116)$ each appears to be an effective independent indicator of poor clinical prognosis in DLBCL. This confirms the results of other studies in similar populations $(3,4,7,10,21,24-28)$. In classifying DLBCL immunohistochemically, a key marker of stratification is expression of the CD10 neutral endopeptidase. Within our cohort, findings 
indicated that positive expression of CD10 was associated with poor median survival time (60 months) as well as longterm overall survival ( $\mathrm{p}=0.0203$ ). A number of studies have demonstrated such an association between CD10 expression and reduced patient survival $(26,29)$, although patient numbers in these studies have often been relatively small. In contrast, various other reports have shown CD10 expression to be associated with improved rather than reduced overall survival $(10,14,29)$. Thus, our findings add to the controversy regarding the prognostic significance of CD10 per se, its cellular role and definitive validity as an independent prognostic marker.

Whilst the search for definitive individual IHC markers of prognosis in DLBCL has often yielded conflicting population dependent results, specific IHC algorithms stratifying these tumours upon the basis of expression of multiple proteins have met with greater success and acceptance. The most prominent of these IHC models/algorithms sub-classify DLBCLs upon the expression of a discrete number of proteins, including Bcl-6, CD10 and MUM-1 (10,21). Each defines tumour 'germinal centre cell phenotype' as being associated with improved overall or event free survival. Conversely, an activated B cell tumour immunophenotype has been associated with reduced survival and poor clinical prognosis. Our data indicated that the GC phenotype as defined conventionally, using CD10 positivity as the primary defining criterion, does not always identify those patients with DLBCL who are at lowest risk of disease progression. This is illustrated by applying 'decision algorithms A and B' to our data. In each instance, in our cohort, the GC phenotype $\left(\mathrm{CD} 10^{+}\right)$was associated with poor clinical prognosis. This led us to propose a third decision algorithm, which distinguishes and defines two apparently distinct groups of GC-like tumours; namely those phenotypically presenting as $\mathrm{CD} 10^{+}$ PP-GC tumours (either: $\mathrm{CD} 10^{+}, \mathrm{Bcl}_{-} 6^{+}, \mathrm{MUM}-1^{-}$or $\mathrm{CD} 10^{+}$, Bcl- $\left.6^{+}, \mathrm{MUM}^{-1}{ }^{+}\right)$and those presenting as GP-GC tumours (CD10- Bcl-6 $6^{+}$MUM-1-). The former PP-GC group of patients showed median survival times of only 11-32 months, as compared to 143 months by the latter group. Thus, the phenotype of the GC cell tumour population that corresponds with the population that presents the least prospect of rapid disease progression is defined as CD10- $\mathrm{Bcl}-6^{+}$MUM- $1^{-}$. Although two groups of GC like tumours were defined a degree of heterogeneity with respect to survival remained apparent within the PP-GC group. This heterogeneity reflected later stage differentiation. Although all PP-GC tumours expressed both CD10 and Bcl-6 those which also expressed MUM-1 were associated with the poorest overall survival. The median survival time observed in this subgroup is almost one third as long as that observed among PP-GC patients presenting tumours which failed to express MUM-1 (11 months c.f. 32 months). This observation supports the concept that MUM-1 is a key transcription factor potentially associated with lymphoma-genesis and tumour progression. This involvement in turn is reflected in patient survival and data supporting this hypothesis, describing downstream MUM-1 targets and MUM-1 polymorphic phenotype, is accumulating from studies of a variety of lymphoid neoplasms. These include investigations targeting lympho-plasmacytic lymphoma, primary effusion lymphoma and multiple myeloma.
The proposed 'Algorithm C' groups DLBCL pragmatically upon the basis of common biomarker expression and patient survival. Thus, PP-GC $\left(\mathrm{CD} 10^{+}\right)$and NGC/ABC (CD10- $\mathrm{Bcl}^{-6^{+}}$ MUM-1 $1^{+}$) tumours defined a patient group which displayed characteristically poor survival. In a similar manner CD10Bcl-6- MUM-1- NGC/ABC and classical CD10- Bcl-6- ${ }^{-}$MUM-1 ${ }^{+}$ $\mathrm{ABC}$ tumours defined a demographic which displayed intermediate survival. Only GP-GC tumours with the CD10Bcl-6 $6^{+}$MUM-1- immunophenotype defined a group of patients who exhibited truly good long-term prognosis. Optimal longterm survival of this subgroup of tumours was also borne out by algorithms $\mathrm{A}$ and $\mathrm{B}$, with the extremes of survival being marked by distinct groups of GC cells displaying opposing survival characteristics. However, the features of the intermediate prognosis group were not so clearly defined by either accepted algorithm. It is interesting to note that classical ABC tumours, considered to be associated with poor prognosis, display low Bcl-6 expression and increased NF- $\mathrm{\kappa B}$ activation (30). In comparison, we observed that $\mathrm{ABC}$ and $\mathrm{NGC/ABC}$ tumours displaying intermediate survival in our cohort did indeed exhibit low Bcl-6 expression. However those NGC/ $\mathrm{ABC}$ tumours displaying worst survival, and grouped together with PP-GC cells, displayed high Bcl-6 expression. This may reflect their preferential activation, but also the potential lack of mutual exclusivity of activation of separate oncogenic pathways in ABC and GC DLBCL cells.

In conjunction with the description and derivation of the IAP-4 specific antibody employed in this study, we have described the importance of the anti-apoptotic protein IAP-4 and Bcl-2 expression in DLBCL (18). Furthermore, we have reported the observation that IAP-4 expression augmented the independent predictive power of $\mathrm{Bcl}-2$ expression in these tumours. In considering the present cohort, expression of Bcl-2 or IAP-4 was significantly associated with reduced survival, in both the defined GP-GC and PP-GC sub-populations. This strengthens observations made independently utilizing commercial IAP-4 specific antibodies $(3,28,31)$. These indicated, that although tumour IAP-4 expression predicted poor survival in DLBCL, this association did not attain significance when GCB and NGCB tumour subgroups were considered independently (28). Such differences may reflect the inconsistency of 'cut off points' applied to define tumour positivity in separate studies, although equally, they may reflect the specificity and spectrum of IAP-4 isoform reactivity of different antibodies. The specificity of the antibody utilized in the present study was confined primarily to the 92Kd anti-apoptotic isoform of IAP-4 (18). These antibodies may be reactive with a broader spectrum of survivin protein isoforms than the antibody employed in the present study, which preferentially recognised the full length anti-apoptotic protein isoform (18). Further expansion of our analysis to explore the prognostic significance of co-expression of both IAP-4 and Bcl-2 in tumour sub-populations was prohibited by virtue of restricted patient numbers. However, trends were evident which support the concept that the balance of antiand pro-apoptotic protein expression may be germane in assembling useful IHC prediction/management models in DLBCL. This suggestion is supported by the observation that resistance to chemotherapy in DLBCL may be linked to upregulation of anti-apoptotic $\mathrm{NF}-\kappa \mathrm{B}$ regulated genes, including 
Bcl-2 and XIAP. The potential importance of investigating such approaches to DLBCL sub-classification has been highlighted in recent publications, which have addressed this subject $(32,33)$. Specific IAP proteins (cIAP1, cIAP2 and XIAP) have been shown to be differentially regulated in a variety of lymphomas, including DLBCL. In studies of the apparent disparate responsiveness of patients with GC and $\mathrm{ABC}$ tumours to combination chemotherapy, three groups of DLBCL were defined upon differential expression of apoptosis related genes (ARG). Of the three ARG groups, two were shown to display poor clinical prognosis: i) an activated apoptosis cascade (AAC) group, presenting evidence of activation of the caspase 9 intrinsic cascade, together with evidence of downstream interference of this same cascade and ii) a cellular cytotoxic response group presenting evidence of activation of the caspase 8 linked death receptor cascades. The third group displaying optimum prognosis was characterised by virtue of lack of expression of those genes characteristic of the other two groups. This definition of good (group iii) and poor prognosis (group ii and i) subsets of DLBCL, agreed only in part with conventional GC and $\mathrm{ABC}$, even though great care was taken in defining $\mathrm{GC} / \mathrm{ABC}$ grouping utilising a 27 gene predictor model adapted from original discriminatory gene signatures proposed in 2003 (34). This supports the suggestion that clinical prognosis may not simply map to GC/ABC classification.

In the search for a means of predicting management strategies for patients with DLBCL, the identification of a single or simple combination of markers may remain elusive. Although, multiple factor models of profiling have been developed and are evolving towards use in the progressive diagnostic laboratory. Currently, such gene expression and modeling systems have produced key expression signatures. These have been honed to incorporate manageable subsets of 13 to 21 vital analytes defining GC-like and ABC-like gene signatures that predict survival in response to combination chemotherapy (35-38). Initially, these signatures appeared somewhat cohort-specific, displaying a lack of consensus. However, further extension of this approach and improved experimental design and standardisation has led to the proposal of a six-gene model able to augment the power of the IPI (LMO2, BCL-6, FN1 gene expression being associated with good clinical prognosis while CCND2, SCYA3 and BCL-2 with poor prognosis) (12). Notably, this model has since been validated in the post-retuximab era (39). The continued evolution of knowledge elucidating the diversity of biology of DLBCL will undoubtedly supplement the ability to establish objective signatures, confirmed by functional studies, which may augment or even supersede both the revised and conventional IPI.

Regardless of advances in automation and standardisation, more sophisticated testing and profiling analyses, despite their power, may for a little while, at least, not see general application in regional and district pathology units. Thus, emphasis may remain on the algorithms, which may be applied routinely in less specialized units utilising relatively conventional IHC techniques. Therefore, if the goal of such a broadly applicable, truly directive yet simple index is to be achieved, studies must be rationalized and standardized rigorously. Notwithstanding the inherent difficulties, attempts are now being made to meet and resolve these problems, unifying techniques and standardising operating procedures. The magnitude of this task ahead, however, has been noted in recent studies performed by the Luenenberg Lymphoma Biomarker Consortium. This work has highlighted the 'variable results and poor reproducibility' of data generated in major laboratories applying semi-quantitative IHC in order to sub-classify DLBCLs (25). However, continuation of this work, combining resources from multiple international trials has defined eight IHC analytes, including Bcl-2 and MUM-1, which proved of greatest predictive potential in the present study, but also including Bcl-6, CD5, HLA-DR, Ki-67 and $\mathrm{CD} 10$. Together it has been proposed that these markers may contribute to a newly proposed 'biological IPI'. However, the robustness of these markers does appear to vary between CHOP and CHOP-R treated patients (40). In the latter group, only BCL-2 and CD-5 were found to fully maintain their predictive power. Thus, it is important to continue this work and appraise the validity of potential biomarkers from the pre- and post-retuximab eras in order to establish a robust and universally applicable 'biological' index.

\section{Acknowledgements}

We would like to extend our thanks to the sponsors of this work, The Bone Marrow and Stem Cell Millennium Fund and Cancer Research North East.

\section{References}

1. A clinical evaluation of the International Lymphoma Study Group classification of non-Hodgkin's lymphoma. The NonHodgkin's Lymphoma Classification Project. Blood 89: 3909-3918, 1997.

2. A predictive model for aggressive non-Hodgkin's lymphoma. The International Non-Hodgkin's Lymphoma Prognostic Factors Project. N Engl J Med 329: 987-994, 1993.

3. Adida C, Haioun C, Gaulard P, Lepage E, Morel P, Briere J, Dombret $\mathrm{H}$, et al: Prognostic significance of survivin expression in diffuse large B-cell lymphomas. Blood 96: 1921-1925, 2000.

4. Barrans SL, Carter I, Owen RG, Davies FE, Patmore RD, Haynes AP, Morgan GJ, et al: Germinal center phenotype and bcl-2 expression combined with the International Prognostic Index improves patient risk stratification in diffuse large B-cell lymphoma. Blood 99: 1136-1143, 2002.

5. Barrans SL, Fenton JA, Banham A, Owen RG and Jack AS: Strong expression of FOXP1 identifies a distinct subset of diffuse large B-cell lymphoma (DLBCL) patients with poor outcome. Blood 104: 2933-2935, 2004.

6. Chang CC, Mcclintock S, Cleveland RP, Trzpuc T, Vesole DH, Logan B, Kajdacsy-Balla A, et al: Immunohistochemical expression patterns of germinal center and activation B-cell markers correlate with prognosis in diffuse large B-cell lymphoma. Am J Surg Pathol 28: 464-470, 2004.

7. Colomo L, Lopez-Guillermo A, Perales M, Rives S, Martinez A, Bosch F, Colomer D, et al: Clinical impact of the differentiation profile assessed by immunophenotyping in patients with diffuse large B-cell lymphoma. Blood 101: 78-84, 2003.

8. Dogan A, Bagdi E, Munson P and Isaacson PG: CD10 and BCL-6 expression in paraffin sections of normal lymphoid tissue and B-cell lymphomas. Am J Surg Pathol 24: 846-852, 2000.

9. Gascoyne RD: Emerging prognostic factors in diffuse large B cell lymphoma. Curr Opin Oncol 16: 436-441, 2004.

10. Hans CP, Weisenburger DD, Greiner TC, Gascoyne RD, Delabie J, Ott G, Muller-Hermelink HK, et al: Confirmation of the molecular classification of diffuse large B-cell lymphoma by immunohistochemistry using a tissue microarray. Blood 103: 275-282, 2004. 
11. Linderoth J, Jerkeman M, Cavallin-Stahl E, Kvaloy S and Torlakovic E: Immunohistochemical expression of CD23 and CD40 may identify prognostically favorable subgroups of diffuse large B-cell lymphoma: a Nordic Lymphoma Group Study. Clin Cancer Res 9: 722-728, 2003.

12. Lossos IS, Czerwinski DK, Alizadeh AA, Wechser MA, Tibshirani R, Botstein D and Levy R: Prediction of survival in diffuse large-B-cell lymphoma based on the expression of six genes. N Engl J Med 350: 1828-1837, 2004.

13. Lossos IS, Jones CD, Warnke R, Natkunam Y, Kaizer H, Zehnder JL, Tibshirani R, et al: Expression of a single gene, BCL-6, strongly predicts survival in patients with diffuse large B-cell lymphoma. Blood 98: 945-951, 2001.

14. Mcclintock S, Perkins S and Cleveland RP: Immunohistochemical expression pattern of germinal centre and activation B-cell markers correlates with prognosis in diffuse large B-cell lymphoma. Mod Pathol 16: A244, 2003.

15. Rosenwald A, Wright G, Chan WC, Connors JM, Campo E, Fisher RI, Gascoyne RD, et al: The use of molecular profiling to predict survival after chemotherapy for diffuse large-B-cell lymphoma. N Engl J Med 346: 1937-1947, 2002.

16. Saez AI, Saez AJ, Artiga MJ, Perez-Rosado A, Camacho FI, Diez A, Garcia JF, et al: Building an outcome predictor model for diffuse large B-cell lymphoma. Am J Pathol 164: 613-622, 2004.

17. Harris NL, Jaffe ES, Stein H, Banks PM, Chan JK, Cleary ML, Delsol G, et al: A revised European-American classification of lymphoid neoplasms: a proposal from the International Lymphoma Study Group. Blood 84: 1361-1392, 1994.

18. Mainou-Fowler T, Overman LM, Dignum H, Wood K, Crosier S, Angus B, Proctor SJ, et al: A new subtype-specific monoclonal antibody for IAP-survivin identifies high-risk patients with diffuse large B-cell lymphoma and improves the prognostic value of bcl-2. Int J Oncol 32: 59-68, 2008.

19. Kaplan GL and Meier P: Non-parametric estimation from incomplete observations. J Am Stat Assoc 53: 457-481, 1958.

20. Cox DR: Regression models and life tables. J R Stat Assoc 34 187-220, 1972.

21. Muris JJ, Meijer CJ, Vos W, Van Krieken JH, Jiwa NM, Ossenkoppele GJ and Oudejans JJ: Immunohistochemical profiling based on Bcl-2, CD10 and MUM1 expression improves risk stratification in patients with primary nodal diffuse large B cell lymphoma. J Pathol 208: 714-723, 2006.

22. Lossos IS and Morgensztern D: Prognostic biomarkers in diffuse large B-cell lymphoma. J Clin Oncol 24: 995-1007, 2006.

23. Ci W, Polo JM and Melnick A: B-cell lymphoma 6 and the molecular pathogenesis of diffuse large B-cell lymphoma. Curr Opin Hematol 15: 381-390, 2008.

24. Reed JC: Bcl-2 family proteins. Oncogene 17: 3225-3236, 1998.

25. De Jong D, Rosenwald A, Chhanabhai M, Gaulard P, Klapper W, Lee A, Sander B, et al: Immunohistochemical prognostic markers in diffuse large B-cell lymphoma: validation of tissue microarray as a prerequisite for broad clinical applications - a study from the Lunenburg Lymphoma Biomarker Consortium. J Clin Oncol 25: 805-812, 2007.

26. Gascoyne RD, Adomat SA, Krajewski S, Krajewska M, Horsman DE, Tolcher AW, O'reilly SE, et al: Prognostic significance of $\mathrm{Bcl}-2$ protein expression and $\mathrm{Bcl}-2$ gene rearrangement in diffuse aggressive non-Hodgkin's lymphoma. Blood 90: 244-251, 1997.
27. Hill ME, Maclennan KA, Cunningham DC, Vaughan Hudson B, Burke M, Clarke P, Di Stefano F, et al: Prognostic significance of BCL-2 expression and bcl-2 major breakpoint region rearrangement in diffuse large cell non-Hodgkin's lymphoma: a British National Lymphoma Investigation Study. Blood 88: 1046-1051, 1996

28. Watanuki-Miyauchi R, Kojima Y, Tsurumi H, Hara T, Goto N, Kasahara S, Saio M, et al: Expression of survivin and of antigen detected by a novel monoclonal antibody, T332, is associated with outcome of diffuse large B-cell lymphoma and its subtypes. Pathol Int 55: 324-330, 2005.

29. Ohshima K, Kawasaki C, Muta H, Muta K, Deyev V, Haraoka S, Suzumiya $\mathrm{J}$, et al: CD10 and Bcl10 expression in diffuse large B-cell lymphoma: CD10 is a marker of improved prognosis. Histopathology 39: 156-162, 2001.

30. Ding BB, Yu JJ, Yu RY, Mendez LM, Shaknovich R, Zhang Y, Cattoretti G, et al: Constitutively activated STAT3 promotes cell proliferation and survival in the activated B-cell subtype of diffuse large B-cell lymphomas. Blood 111: 1515-1523, 2008.

31. Kuttler F, Valnet-Rabier MB, Angonin R, Ferrand C, Deconinck E, Mougin C, Cahn JY, et al: Relationship between expression of genes involved in cell cycle control and apoptosis in diffuse large B cell lymphoma: a preferential survivin-cyclin B link. Leukemia 16: 726-735, 2002.

32. Akyurek N, Ren Y, Rassidakis GZ, Schlette EJ and Medeiros LJ: Expression of inhibitor of apoptosis proteins in B-cell nonHodgkin and Hodgkin lymphomas. Cancer 107: 1844-1851, 2006.

33. Muris JJ, Ylstra B, Cillessen SA, Ossenkoppele GJ, KluinNelemans JC, Eijk PP, Nota B, et al: Profiling of apoptosis genes allows for clinical stratification of primary nodal diffuse large B-cell lymphomas. Br J Haematol 136: 38-47, 2007.

34. Wright G, Tan B, Rosenwald A, Hurt EH, Wiestner A and Staudt LM: A gene expression-based method to diagnose clinically distinct subgroups of diffuse large B cell lymphoma. Proc Natl Acad Sci USA 100: 9991-9996, 2003.

35. Alizadeh AA, Eisen MB, Davis RE, Ma C, Lossos IS, Rosenwald A, Boldrick JC, et al: Distinct types of diffuse large B-cell lymphoma identified by gene expression profiling. Nature 403: 503-511, 2000.

36. Rosenwald A and Staudt LM: Clinical translation of gene expression profiling in lymphomas and leukemias. Semin Oncol 29: 258-263, 2002

37. Shipp MA, Ross KN, Tamayo P, Weng AP, Kutok JL, Aguiar RC, Gaasenbeek M, et al: Diffuse large B-cell lymphoma outcome prediction by gene-expression profiling and supervised machine learning. Nat Med 8: 68-74, 2002.

38. Woehrer S, Hejna M, Skrabs C, Drach J, Zielinski CC, Jaeger U and Raderer M: Rituximab, Ara-C, dexamethasone and oxaliplatin is safe and active in heavily pretreated patients with diffuse large B-cell lymphoma. Oncology 69: 499-502, 2005

39. Malumbres R, Chen J, Tibshirani R, Johnson Na, Sehn LH, Natkunam Y, Briones J, et al: Paraffin-based 6-gene model predicts outcome in diffuse large B-cell lymphoma patients treated with R-CHOP. Blood 111: 5509-5514, 2008.

40. Consortium Llb: First results of an international study to establish a new clinico-biological prognostic index for Diffuse Large B Cell Lymphoma (DLBCL). Ann Oncol 19: abs. 054bis, 2008. 